\title{
Pro-atrial natriuretic peptide and pro-vasopressin for predicting short-term and long-term survival in community-acquired pneumonia: results from the German Competence Network CAPNETZ
}

\author{
Stefan Krüger, ${ }^{1}$ Santiago Ewig, ${ }^{2}$ Jan Kunde, ${ }^{3}$ Oliver Hartmann, ${ }^{3}$ Norbert Suttorp, ${ }^{4}$ \\ Tobias Welte, ${ }^{5}$ the CAPNETZ Study Group
}

\begin{abstract}
${ }^{1}$ Medical Clinic I, Medical Faculty, RWTH University Aachen, Germany

${ }^{2}$ Thoraxzentrum Ruhrgebiet, Kliniken für Pneumologie und Infektiologie, Ev. Krankenhaus Herne und Augusta Kranken-Anstalt Bochum, Germany

${ }^{3}$ Research Department, BRAHMS AG, Hennigsdorf, Germany

${ }^{4}$ Department of Internal Medicine/Infectious Diseases and Pulmonary Medicine, Charite-University Medicine, Berlin, Germany ${ }^{5}$ Department of Pneumology, Hannover Medical School, University Clinic, Hannover, Germany
\end{abstract}

\section{Correspondence to}

Professor Dr med Tobias Welte, Department of Pneumology, Hannover Medical School, University Clinic, Carl Neuberg Str. 1, D-30625 Hannover Germany;

welte.tobias@mh-hannover.de

Received 5 June 2009

Accepted 19 December 2009

\begin{abstract}
Background Community-acquired pneumonia (CAP) is the most important clinical infection with a high longterm mortality rate. The aim of this study was to evaluate the value of biomarkers for the prediction of short-term and long-term mortality in CAP.

Methods A total of 1740 patients of mean \pm SD age $60 \pm 18$ years ( $45 \%$ female) with proven CAP were enrolled in the study. Mid-regional pro-atrial natriuretic peptide (MR-proANP), C-terminal pro-atrial vasopressin (CT-proAVP), procalcitonin , C-reactive protein, leucocyte count (WBC) and CRB-65 score were determined on admission. Patients were followed up for 180 days.

Results MR-proANP and CT-proAVP levels increased with increasing severity of CAP, classified according to CRB-65 score. In patients who died within 28 and 180 days, median MR-proANP (313.9 vs 80.0 and 277.8 vs $76.0 \mathrm{pmol} / \mathrm{l}$, each $\mathrm{p}<0.0001$ ) and CT-proAVP (42.6 vs 11.2 and 33.2 vs $10.7 \mathrm{pmol} / \mathrm{l}$, each $p<0.0001$ ) levels were significantly higher than the levels in survivors. In receiver operating characteristics analysis for survival at 28 and 180 days, the areas under the curves (AUCs) for CT-proAVP $(0.84,95 \% \mathrm{Cl} 0.82$ to 0.86 and $0.78,95 \% \mathrm{Cl}$ 0.76 to 0.80$)$ and MR-proANP $(0.81,95 \% \mathrm{Cl} 0.79$ to 0.83 and $0.81,95 \% \mathrm{Cl} 0.79$ to 0.83 ) were superior to the AUC of CRB-65 $(0.74,95 \% \mathrm{Cl} 0.71$ to 0.76 and $0.71,95 \% \mathrm{Cl}$ 0.69 to $0.74, p<0.05)$, procalcitonin, $C$-reactive protein and WBC. In multivariable Cox proportional hazards regression analyses adjusted for comorbidity and pneumonia severity, MR-proANP and CT-proAVP were independent and the strongest predictors of short-term and long-term mortality.
\end{abstract}

Conclusions MR-proANP and CT-proAVP are powerful tools for the prediction of short-term and long-term risk stratification of patients with CAP.

\section{INTRODUCTION}

Community-acquired pneumonia (CAP) is the most common potentially fatal infectious disease throughout the western industrialised countries. ${ }^{12}$ The established CAP risk scores-the pneumonia severity index (PSI) and the CURB score and its modifications-are validated for the prediction of short-term mortality only. ${ }^{3-7}$ However, several studies have shown that patients surviving CAP have a subsequent mortality rate substantially higher than expected from an age-matched control population. $^{8-11}$ Only a limited number of larger studies have examined long-term mortality in CAP and its risk factors. ${ }^{8-11}$ A validated score for the determination of long-term mortality after CAP is not available.

Inflammatory markers such as C-reactive protein (CRP) and procalcitonin (PCT) have been shown to be predictive of short-term (usually 28-day or 30day) mortality. ${ }^{12}$ We have recently shown in a pilot study that C-terminal pro-atrial vasopressin (CTproAVP) and mid-regional pro-atrial natriuretic peptide (MR-proANP) are also good predictors of short-term mortality. ${ }^{13}$ CT-proAVP and MRproANP levels are significantly lower in short-term survivors of CAP than in those who die within 28 days, and they correlate with the severity of the disease as measured by the CRB-65 score.

This study was undertaken to investigate the predictive potential of MR-proANP and CT-proAVP for long-term mortality in patients with CAP.

\section{METHODS}

\section{Patients}

CAPNETZ represents a German Competence network for the study of CAP (CAPNETZ, http:// www.capnetz.de). ${ }^{1}$ Within CAPNETZ, all new cases of CAP are reported via a network of sentinel practices and hospitals to the study monitor of the corresponding local clinical centre. Inclusion criteria are age $\geq 18$ years, a pulmonary infiltrate diagnosed by chest $\mathrm{x}$-ray, clinical symptoms consisting of cough or purulent sputum or positive auscultation. Exclusion criteria include age $<18$ years, acquired or therapeutically-induced immune deficiency, active tuberculosis or possible nosocomial acquisition of infection (hospitalisation $<4$ weeks before infection). After inclusion in CAPNETZ, all clinical parameters of the patients are stored in an electronic database

The CRB-65 score was determined in all patients The score consists of four variables: confusion, respiratory rate $\geq 30 / \mathrm{min}$, systolic blood pressure $<90 \mathrm{mmHg}$ or diastolic blood pressure $\leq 60 \mathrm{~mm} \mathrm{Hg}$, age $\geq 65$ years. ${ }^{4}{ }^{14}$ One point is given for each parameter present which results in CRB-65 scores of $0-4$. The microbiological investigation in patients in CAPNETZ has been described previously. ${ }^{1}$ Briefly, it included sputum samples with Gram stain and culture, blood samples for serological testing for Chlamydophila pneumoniae and Mycoplasma pneumoniae and a urine sample for the detection of Legionella pneumophila and Streptococcus pneumoniae. Detection of respiratory pathogens was 
performed according to standard methods and established microbiological guidelines. ${ }^{15}$

All patients were assessed at first presentation and during follow-up according to a standardised data sheet. After 14 days, all patients or relatives were contacted personally or by telephone for a structured interview on outcome parameters including death. To assess the number of pneumonia-related deaths within 28 days and 180 days, the interview was repeated for all patients after 180 days. Short-term mortality was defined as death within the first 28 days. Long-term mortality was defined as death from day 29 to 180 days after inclusion in the study.

\section{Laboratory measurements}

Venous blood samples were collected at the time of inclusion in the study. Serum CRP was measured by nephelometry with a commercially available assay (Behring Diagnostics, Marburg, Germany). Serum PCT was determined by an immunofluorescent assay (BRAHMS PCT sensitive KRYPTOR, BRAHMS AG, Henningsdorf, Germany).

Because of the short half-life of atrial natriuretic peptide (ANP) and atrial vasopressin (AVP) as well as the instability and platelet binding of AVP, precursor fragments of both hormones (mid-regional pro-atrial natriuretic peptide (MR-proANP) and Cterminal pro-atrial vasopressin (CT-proAVP; Copeptin) were analysed as alternative diagnostic targets. MR-proANP and CTproAVP levels directly reflect the release of their rapidly degraded active hormones ANP and AVP. ${ }^{16-19}$

MR-proANP measurements were performed using an immunoluminometric sandwich assay (BRAHMS MR-proANP LIA, BRAHMS AG) as described in detail elsewhere. ${ }^{16}{ }^{19}$ CT-proAVP measurements were performed with a new sandwich immunoluminometric assay, as described recently. ${ }^{17}$ Briefly, two polyclonal antibodies to the C-terminal region (covering amino acids 132-164 of pre-proAVP) were used. Laboratory measurements were performed in a blinded fashion without knowledge of clinical parameters and microbiological results. Measurements of PCT, CT-proAVP and MR-proANP were performed in one central laboratory.

\section{Statistical analysis}

Data were statistically analysed with Graph Pad Prism 4.0 (GraphPad Software Inc) and MedCalc 9.0.1.0 package (MedCalc software). Distribution of the analysed continuous variables (eg, scores, biomarkers) for normality was tested with the Kolmogorov-Smirnov test. To compare inpatients and outpatients as well as short-term/long-term survivors and deaths, two group comparisons of continuous variables were performed by the Mann-Whitney U test. Categorical data were compared using the $\chi^{2}$ test. For comparisons of (continuous) marker values and pneumonia severity (CRB-65 class), Kruskal-Wallis one-way analysis of variance was used. Correlation analyses were performed using Spearman rank correlation. To compare the accuracy of markers to predict hospitalisation and to identify best cut-off concentrations to predict the risk of mortality, we constructed receiver operating characteristic curves and determined the area under the curve (AUC). Using the identified cutoff concentrations to predict mortality risk, Kaplan-Meier survival curves were generated to visualise the distribution of times from baseline to death, and the log-rank test was performed to compare the survival curves between groups. ORs were calculated from cross tabulation of markers above/below the cut-off values and outcome. Furthermore, Cox proportional hazards regression was used to analyse the effect of the biomarkers on survival in univariate analyses. To show that the proposed markers are independent of the known risk factors CRB-65, presence of congestive heart failure or comorbidities, multivariable Cox regression analyses were performed. Comorbidities were defined as the presence of one or more of the following: congestive heart failure, chronic obstructive pulmonary disease (COPD), chronic renal disease, chronic liver disease, cerebrovascular disease, malignancy or diabetes mellitus. Proportional hazards assumption for the Cox regression was tested based on weighted residuals. None of the time-dependent coefficients $\beta(\mathrm{t})$ deviated significantly from a straight line. Positive and negative predictive values were calculated to give information about the probability that the new biomarkers will give the correct diagnosis-in our case, the event of mortality. All statistical tests were two-tailed and a $\mathrm{p}$ value $<0.05$ was considered statistically significant.

\section{RESULTS \\ Patients}

The study population comprised 1740 patients with a mean age of $60 \pm 18$ years (range 18-98) and $55 \%$ were male; $65 \%$ of patients were hospitalised and $35 \%$ were treated as outpatients. The causative pathogen was found in 331 patients $(19.0 \%)$ (typical pyogenic bacterial infection, $\mathrm{n}=100$ (5.7\%); atypical bacterial infection (Legionella spp, Mycoplasma and C pneumoniae), $\mathrm{n}=170$ (9.8\%); viral infection, $\mathrm{n}=21(1.2 \%)$; mixed infections with two or more pathogens, $n=40(2.3 \%))$. Overall, $58.8 \%$ of patients were characterised by one or more relevant coexisting illnesses, including congestive heart failure, COPD, chronic renal disease, chronic liver disease, cerebrovascular disease, cancer and diabetes mellitus. Comorbidities and abnormal physical examination findings were more frequent in hospitalised patients than in outpatients (table 1).

\section{Outcome}

Of the 1740 patients included in the study, four patients $(0.2 \%)$ were lost to follow-up. Seventy-eight patients (4.5\%) died within 28 days and an additional 93 patients (5.6\%) died between days 29 and 180. Overall, 171 patients (9.9\%) died within 180 days. The proportion of short-term deaths of outpatients was significantly lower than that of hospitalised patients (1 $(0.2 \%)$ vs $77(6.9 \%), p<0.0001)$. The isolated proportion of long-term deaths of outpatients was significantly lower than that of hospitalised patients $(3(0.5 \%)$ vs $90(8.6 \%), p<0.0001)$. One hundred and five of the 1736 patients (6\%) were from nursing home/long-term care facilities. The overall number of deaths at 180 days of patients from nursing home/long-term care facilities was higher than of patients who were not from nursing home/long-term care facilities (23 (22\%) vs 148 (9.1\%), p<0.01).

\section{Pneumonia severity}

Severity of pneumonia was assessed using the CRB-65 score. In outpatients, pneumonia severity assessed by CRB-65 points was lower than in hospitalised patients $(p<0.0001)$. MR-proANP and CT-proAVP levels increased with increasing severity of CAP $(p<0.0001)$. CRP, WBC and PCT levels were also associated with increasing pneumonia severity $(\mathrm{p}<0.0001)$.

\section{Prediction of short-term clinical outcome}

In patients who died within 28 days of follow-up, MR-proANP and CT-proAVP levels on admission were significantly higher than levels in survivors (median (IOR) MR-proANP 313.9 $(154.0-615.6)$ vs $80.0 \quad(47.0-175.0) \mathrm{pmol} / \mathrm{l}, \quad \mathrm{p}<0.0001$; CT-proAVP $42.6 \quad(20.6-75.8)$ vs $11.2 \quad(5.1-20.7) \mathrm{pmol} / \mathrm{l}$, $\mathrm{p}<0.0001$; figure 1$)$. The respective values for CRP were 155.0 
Table 1 Baseline characteristics of patients

\begin{tabular}{|c|c|c|c|c|}
\hline Characteristic & $\begin{array}{l}\text { Total } \\
(\mathrm{n}=1740)\end{array}$ & $\begin{array}{l}\text { Inpatients } \\
(\mathrm{n}=1131)\end{array}$ & $\begin{array}{l}\text { Outpatients } \\
\text { (n = 609) }\end{array}$ & p Value* \\
\hline Mean $\pm S D$ age (years) & $60 \pm 18$ & $64 \pm 18$ & $51 \pm 16$ & $<0.0001$ \\
\hline Male sex, n (\%) & $961(55.2)$ & $682(60.3)$ & $279(45.8)$ & $<0.0001$ \\
\hline Smokers, n (\%) & $513(29.5)$ & $329(29.1)$ & $184(30.2)$ & 0.6 \\
\hline \multicolumn{5}{|l|}{ Comorbidities, n (\%) } \\
\hline Congestive heart failure & $298(17.1)$ & $262(23.2)$ & $36(5.9)$ & $<0.0001$ \\
\hline COPD & $610(35.1)$ & $432(38.2)$ & $178(29.2)$ & 0.0002 \\
\hline Chronic renal disease & $141(8.1)$ & $132(11.7)$ & $9(1.5)$ & 0.0001 \\
\hline Chronic liver disease & $57(3.3)$ & $41(3.7)$ & $16(2.6)$ & 0.3 \\
\hline Cerebrovascular disease & $165(9.5)$ & $157(13.9)$ & $8(1.3)$ & $<0.0001$ \\
\hline Malignancy & $166(9.5)$ & $128(11.3)$ & $38(6.2)$ & 0.0006 \\
\hline Diabetes mellitus & $286(16.4)$ & $247(21.9)$ & $39(6.4)$ & $<0.0001$ \\
\hline \multicolumn{5}{|l|}{ Physical examination findings, $\mathrm{n}(\%)$} \\
\hline Confusion & $143(8.2)$ & $132(11.7)$ & $11(1.8)$ & $<0.0001$ \\
\hline Respiratory rate $\geq 30 / \mathrm{min}$ & $146(8.4)$ & $127(11.2)$ & $19(3.1)$ & 0.0001 \\
\hline $\begin{array}{l}\text { Blood pressure } \\
\text { systolic }<90 \mathrm{~mm} \mathrm{Hg} \text { or diastolic } \leq 60 \mathrm{~mm} \mathrm{Hg}\end{array}$ & $311(17.9)$ & $251(22.2)$ & $60(9.9)$ & $<0.0001$ \\
\hline \multicolumn{5}{|l|}{ Aetiology of CAP, n (\%) } \\
\hline Typical bacterial pathogens $\uparrow$ & $100(5.7)$ & $74(6.5)$ & $26(4.3)$ & 0.05 \\
\hline Atypical pathogens $\ddagger$ & $170(9.8)$ & $100(9.6)$ & $61(10.0)$ & 0.4 \\
\hline Viruses $\S$ & $21(1.2)$ & $13(1.1)$ & $8(1.3)$ & 0.8 \\
\hline Mixed 9 & $40(2.3)$ & $31(2.7)$ & $9(1.5)$ & 0.09 \\
\hline Unknown & $1409(81.0)$ & 904 (79.9) & $505(82.9)$ & 0.1 \\
\hline Prior antibiotics, n (\%) & $480(27.6)$ & $279(24.7)$ & $201(33.0)$ & 0.0002 \\
\hline \multicolumn{5}{|l|}{ CRB-65, n (\%) } \\
\hline 0 & $726(41.7)$ & $354(31.3)$ & $372(61.1)$ & $<0.0001$ \\
\hline 1 & 717 (41.2) & $506(44.7)$ & $211(34.6)$ & $<0.0001$ \\
\hline 2 & $232(13.3)$ & $207(18.3)$ & $25(4.1)$ & $<0.0001$ \\
\hline 3 & $58(3.3)$ & $57(5.0)$ & $1(0.2)$ & $<0.0001$ \\
\hline 4 & $7(0.4)$ & $7(0.6)$ & $0(0)$ & 0.05 \\
\hline 28-day mortality, n (\%) & $78(4.5)$ & $77(6.9)$ & $1(0.2)$ & $<0.0001$ \\
\hline Cumulative 180-day mortality, n (\%) & $171(9.9)$ & $167(14.8)$ & $4(0.7)$ & $<0.0001$ \\
\hline
\end{tabular}

*Comparison of inpatients vs outpatients.

†S pneumoniae, H influenzae, E coli, $S$ aureus, Moraxella catarrhalis, Enterobacter spp, Proteus mirabilis, Pseudomonas spp, Citrobacter spp, Enterococcus faecium, Klebsiella pneumoniae, Streptococcus agalactiae.

$\ddagger$ Mycoplasma pneumoniae, Legionella pneumophila, Chlamydophila pneumoniae.

SInfluenza A, respiratory syncytial virus.

qInfections with two or more pathogens including the following combinations: two or more typical or atypical bacteria, typical bacterial plus atypical bacterial infection, typical or atypical bacterial infection plus viruses.

$\mathrm{CAP}$, community-acquired pneumonia; COPD, chronic obstructive pulmonary disease.

$(69.1-296.5)$ vs $72.5(21.0-176.9) \mathrm{mg} / \mathrm{l}(\mathrm{p}<0.0001)$ and for PCT $0.83(0.19-4.49)$ vs $0.10(0.06-0.35) \mathrm{ng} / \mathrm{ml}(\mathrm{p}<0.0001)$.

The AUCs for short-term mortality of CT-proAVP $(0.84,95 \%$ CI 0.82 to 0.86 ) and MR-proANP (0.81, 95\% CI 0.79 to 0.83 ) were significantly higher than those of PCT $(0.76,95 \%$ CI 0.73 to $0.78 ; \mathrm{p}=0.02)$, CRB-65 $(0.74,95 \%$ CI 0.71 to $0.76, p=0.02)$, $\operatorname{CRP}(0.67,95 \%$ CI 0.65 to $0.69, \mathrm{p}<0.001)$ and WBC $(0.60,95 \% \mathrm{CI}$ 0.57 to $0.62, \mathrm{p}<0.001$ ) (figure $2 \mathrm{~A}$ ).

The optimal threshold (minimal false negative and false positive results) to predict short-term death was $110 \mathrm{pmol} / 1$ for MR-proANP with a sensitivity of $91 \%$, specificity of $62 \%$, positive predictive value of $10 \%$ and negative predictive value of $99 \%$ (OR 14.6, 95\% CI 7.0 to 30.5). The optimal threshold for CTproAVP to predict death was $29.0 \mathrm{pmol} / 1$ with a sensitivity of $68 \%$, specificity of $86 \%$, positive predictive value of $19 \%$ and negative predictive value of $98 \%$ (OR 13.1, 95\% CI 7.8 to 22.2).

\section{Prediction of long-term clinical outcome}

In patients who died during 180 days of follow-up, MR-proANP and CT-proAVP levels on admission were significantly higher than in survivors (median (IOR) MR-proANP 277.8 $(138.0-626.5) \quad$ vs $76.0 \quad(45.7-161.6) \mathrm{pmol} / \mathrm{l}, \quad \mathrm{p}<0.0001$;
CT-proAVP $33.2 \quad(16.4-60.4)$ vs $10.7 \quad(5.0-19.7) \mathrm{pmol} / \mathrm{l}$, $\mathrm{p}<0.0001$; figure 1). The respective values for CRP were 101.7 (39.5-215.3) vs $71.8(20.0-180.0) \mathrm{mg} / \mathrm{l}(\mathrm{p}=0.0004)$ and for PCT $0.43(0.13-1.89)$ vs $0.09(0.06-0.32) \mathrm{ng} / \mathrm{ml}(\mathrm{p}<0.0001)$.

For cumulative long-term mortality at 180 days, AUC was highest for MR-proANP $(0.81,95 \%$ CI 0.79 to 0.83$)$ which was comparable to the AUC of CT-proAVP $(0.78,95 \%$ CI 0.76 to 0.80 ; $\mathrm{p}=\mathrm{NS})$. The AUCs for PCT $(0.70,95 \%$ CI 0.67 to $0.72 ; \mathrm{p}<0.001)$ CRB-65 $(0.71,95 \%$ CI 0.69 to $0.74, p=0.001)$, CRP $(0.57,95 \% \mathrm{CI}$ 0.55 to $0.60, \mathrm{p}<0.001)$ and $\mathrm{WBC}(0.61,95 \%$ CI 0.59 to 0.64 , $\mathrm{p}<0.001)$ were significantly lower compared with MR-proANP and CT-proAVP (figure $2 \mathrm{~B}$ ).

The optimal threshold (minimal false negative and false positive results) to predict death at 180 days was $102 \mathrm{pmol} / \mathrm{l}$ for MR-proANP with a sensitivity of $88 \%$, specificity of $62 \%$, positive predictive value of $20 \%$ and negative predictive value of $98 \%$ (OR 11.9, 95\% CI 7.4 to 19.0). The optimal threshold for CTproAVP to predict death was $22.3 \mathrm{pmol} / 1$ with a sensitivity of $65 \%$, specificity of $80 \%$, positive predictive value of $26 \%$ and negative predictive value of $95 \%$ (OR 7.3, 95\% CI 5.2 to 10.4). Figure 3 shows the Kaplan-Meier curves according to three different biomarkers. 
Figure 1 Admission levels of (A) midregional pro-atrial natriuretic peptide (MR-proANP) and (B) C-terminal proatrial vasopressin (CT-proAVP) in patients with community-acquired pneumonia (CAP) according to outcome (28-day and 180-day mortality). Solid lines denote median values, boxes represent 25th to 75th percentiles and whiskers indicate the range.
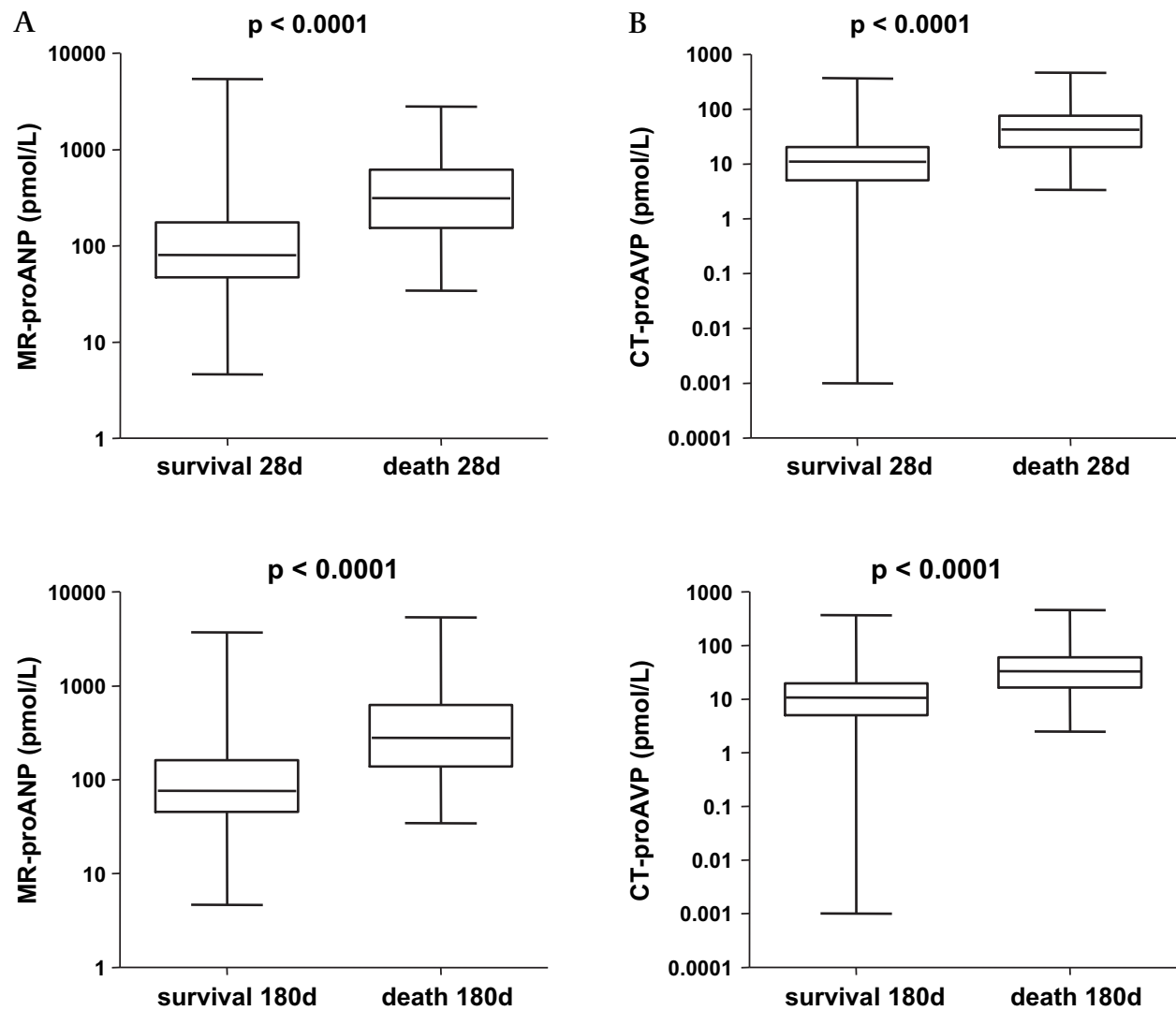

In univariate Cox proportional hazards regression analysis, increased MR-proANP and CT-proAVP levels had significant risk ratios independent of confounders such as the presence of comorbidity and pneumonia severity (table 2). In multivariable Cox proportional hazards regression analyses adjusted for these confounders, the strongest predictors of short-term and cumulative long-term mortality were serum MR-proANP and CTproAVP levels $>110 \mathrm{pmol} / \mathrm{l}$ and $29.0 \mathrm{pmol} / 1$ for short-term mortality, respectively, and MR-proANP and CT-proAVP levels $>102 \mathrm{pmol} / \mathrm{l}$ and $22.3 \mathrm{pmol} / \mathrm{l}$ for death at 180 days. Highest risk ratios were calculated for MR-proANP.

\section{DISCUSSION}

The main findings of this study are (1) MR-proANP, CT-proAVP and PCT serum levels increase with increasing pneumonia severity as assessed by CRB-65 score; (2) MR-proANP and CT-proAVP levels are good predictors of 28-day and 180-day mortality from pneumonia; (3) MR-proANP and CT-proAVP are significantly better predictors for 28-day mortality and especially for 180-day mortality than the CRB-65 score and PCT, CRP and WBC.

Currently, two severity prediction tools have been developed to assess pneumonia severity at initial presentation. These include the PSI and the CURB score and its modifications (CURB-65, CRB-65). ${ }^{3} 4720$ Risk assessment by the CRB-65 score yielded comparable results to the CURB or PSI score in patients with CAP. ${ }^{14}$ More recently, biomarkers have emerged as predictors of pneumonia severity. In previous studies we showed that the biomarkers PCT, MR-proANP and CT-proAVP correlated with severity and short-term mortality. ${ }^{12}{ }^{13}$ In a smaller study of 373 patients with lower respiratory tract infections, MR-proANP levels were significantly higher in short-term non-survivors than in survivors. ${ }^{21} \mathrm{~A}$ further recent study showed that MR-proANP
Figure 2 Receiver operator characteristic curves for mid-regional pro-atrial natriuretic peptide (MRproANP), C-terminal pro-atrial vasopressin (CT-proAVP), procalcitonin (PCT), C-reactive protein (CRP), white blood cells (WBC) and CRB-65 score with respect to prediction of mortality within (A) 28 days and (B) 180 days.
A

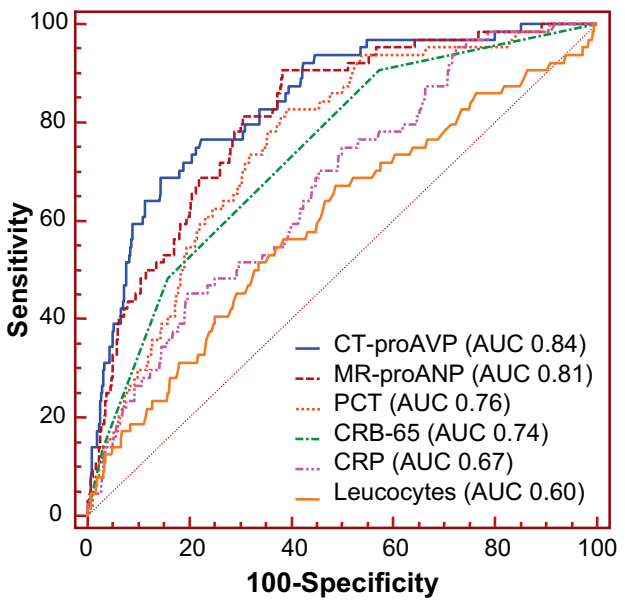

B

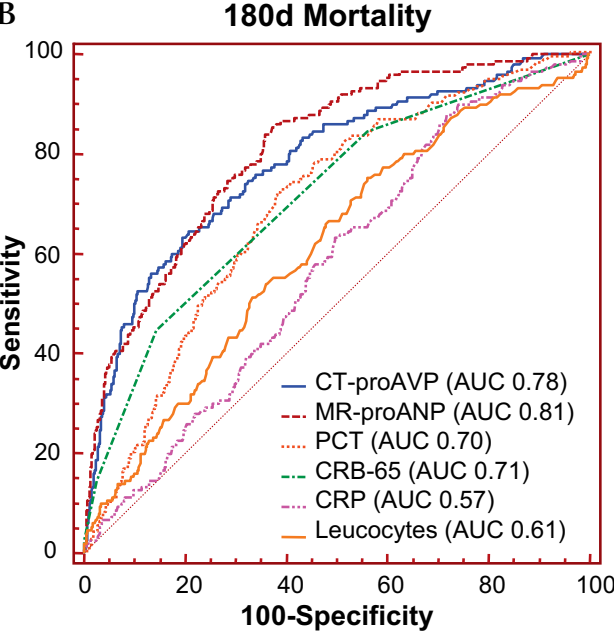


Figure 3 Kaplan-Meier analysis according to $(A)$ mid-regional pro-atrial natriuretic peptide (MR-proANP levels) (groups below ( $n=998$, solid line) and above ( $n=727$, dotted line) a cut-off concentration of $102 \mathrm{pmol} / \mathrm{l} ; \mathrm{p}<0.0001$, log-rank test); (B) C-terminal pro-atrial vasopressin (CT-proAVP) levels (groups below ( $n=1270$, solid line) and above ( $\mathrm{n}=391$, dotted line) a cut-off concentration of $22.3 \mathrm{pmol} / \mathrm{l}$; $\mathrm{p}<0.0001$, log-rank test); (C) procalcitonin (PCT) levels (groups below ( $n=1037$, solid line) and above $(n=688$, dotted line) a cut-off concentration of $0.15 \mathrm{ng} / \mathrm{ml} ; \mathrm{p}<0.0001$, log-rank test).
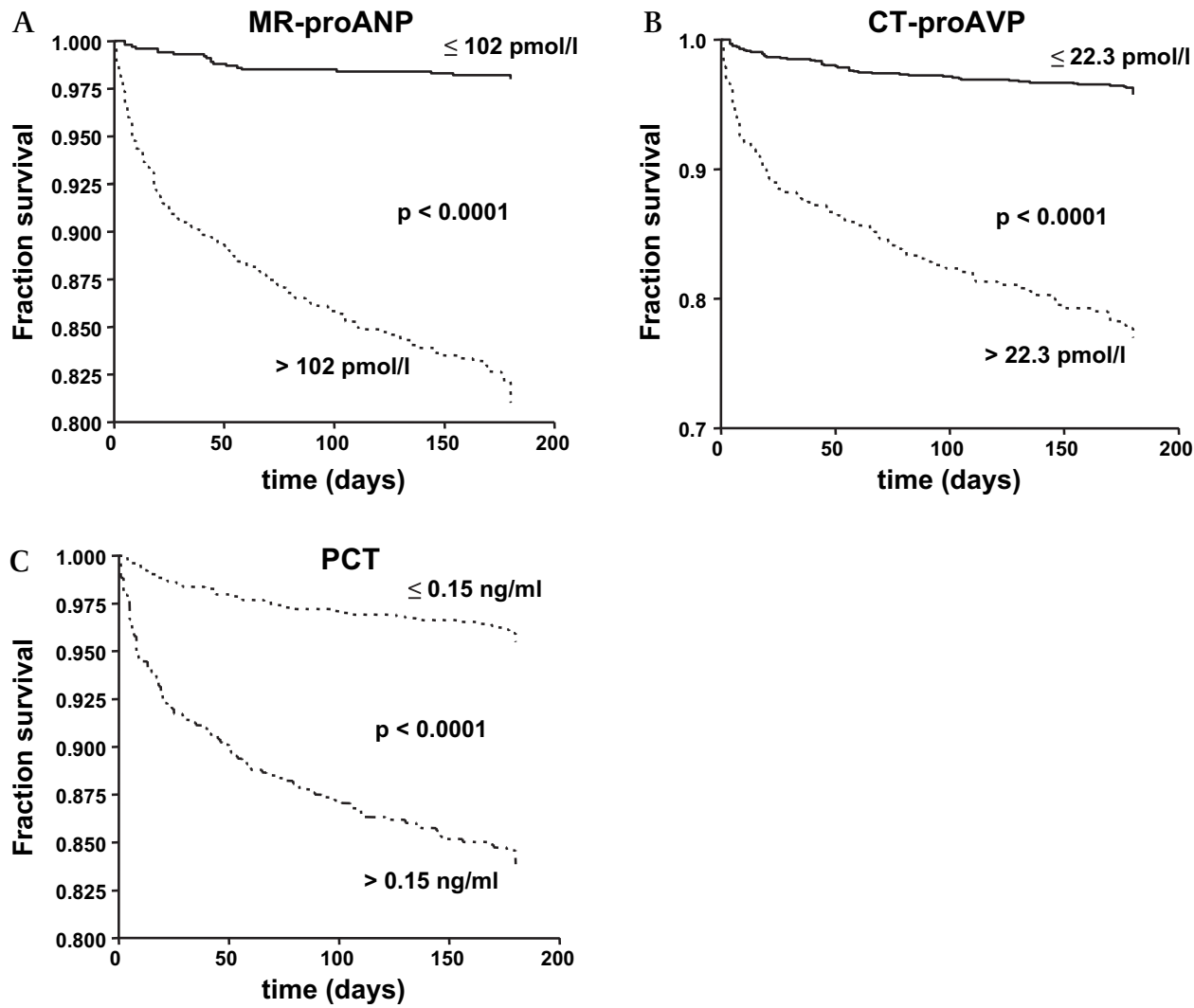

levels increase with severity of CAP and are higher in patients who develop complications or die. ${ }^{22}$ In the present study the association of biomarkers such as MR-proANP, CT-proAVP and PCT with pneumonia severity and its high predictive potential for short-term mortality was confirmed. Interestingly, MRproANP and CT-proAVP performed even better than the CRB-65 score as assessed by receiver operating characteristic curves. A

Table 2 Results of Cox proportional hazards regression analysis of the effect of baseline variables on long-term survival; outcome is death within follow-up of 180 days, patients lost to follow-up were censored

\begin{tabular}{|c|c|c|}
\hline Baseline variables & HR $(95 \% \mathrm{CI})$ & p Value \\
\hline \multicolumn{3}{|l|}{ Univariate analysis } \\
\hline MR-proANP (>102 pmol/l) & $10.98(6.90$ to 17.47$)$ & $<0.0001$ \\
\hline CT-proAVP (>22.3 pmol/l) & 6.47 (4.69 to 8.94$)$ & $<0.0001$ \\
\hline CRB-65 $(>1)$ & 4.25 (3.14 to 5.77$)$ & $<0.0001$ \\
\hline Congestive heart failure & $3.67(2.70$ to 4.99$)$ & $<0.0001$ \\
\hline Comorbidity* & $7.62(4.50$ to 12.92$)$ & $<0.0001$ \\
\hline \multicolumn{3}{|c|}{ Multivariate Cox regression model 1} \\
\hline MR-proANP (>102 pmol/l) & $7.67(4.72$ to 12.45$)$ & $<0.0001$ \\
\hline CRB-65 $(>1)$ & 2.43 (1.78 to 3.32$)$ & $<0.0001$ \\
\hline Congestive heart failure & $1.64(1.19$ to 2.25$)$ & 0.002 \\
\hline \multicolumn{3}{|c|}{ Multivariate Cox regression model 2} \\
\hline MR-proANP (>102 pmol/l) & 6.37 (3.92 to 10.35$)$ & $<0.0001$ \\
\hline CRB-65 $(>1)$ & $2.28(1.67$ to 3.11$)$ & $<0.0001$ \\
\hline Comorbidity* & $3.23(1.87$ to 5.59$)$ & $<0.001$ \\
\hline \multicolumn{3}{|c|}{ Multivariate Cox regression model 3} \\
\hline CT-proAVP (>22.3 pmol/l) & $4.43(3.18$ to 6.17$)$ & $<0.0001$ \\
\hline CRB-65 $(>1)$ & $2.38(1.74$ to 3.25$)$ & $<0.0001$ \\
\hline Comorbidity* & 4.65 (2.71 to 7.96$)$ & $<0.0001$ \\
\hline
\end{tabular}

*Presence of one or more comorbidities: congestive heart failure, chronic obstructive pulmonary disease, chronic renal disease, chronic liver disease, cerebrovascular disease, malignancy, or diabetes mellitus.

CT-proAVP, C-terminal pro-atrial vasopressin; MR-proANP, mid-regional pro-atrial natriuretic peptide. new finding of this study is that MR-proANP and CT-proAVP show a high predictive value for long-term mortality as well as for short-term mortality. Although the positive predictive values are low, the very high negative predictive potential of MRproANP and CT-proAVP for short-term as well as long-term mortality might be useful for identifying patients who can be safely treated despite an increased CRB-65 score. Patients with raised levels of MR-proANP and CT-proAVP should be examined with respect to underlying relevant cardiovascular morbidity.

Several investigations have consistently shown that long-term mortality is excessive after an acute phase of CAP compared with an age-matched cohort. ${ }^{8} 923$ The highest impact of CAP on long-term mortality is in the first year after the initial episode of CAP, but the association with excess long-term mortality could be demonstrated for at least 5 years. Independent clinical predictors of long-term mortality are increasing age, cardiovascular and cerebrovascular comorbidities and altered mental state. Acute physiological changes are not helpful for determining longterm mortality; they are associated with short-term mortality only. ${ }^{23}$ The main causes of death after an episode of CAP are cardiovascular diseases and cancer, but chronic lower respiratory disease, renal failure and infection also have an important role. ${ }^{23} 24$ Subjects with significant comorbidities such as chronic heart disease, lung disease, renal disease or cancer have higher circulating inflammatory marker concentrations and are more likely to die of the underlying comorbidity. Yende et al found that persistent inflammation, defined as increased circulating levels of interleukin-6 (IL6) and IL10 at hospital discharge after CAP were associated with all-cause and cause-specific mortality over 1 year despite the resolution of clinical signs of an acute infection. ${ }^{24}$ At hospital discharge more than half the patients had raised serum concentrations of IL6. Interestingly, they found that in many patients who died of cardiovascular disease, cancer and renal failure long-term after CAP, the presence of these 
underlying diseases was unknown before the occurrence of CAP and even after the CAP episode. This supports the traditional hypothesis that, in many cases (especially elderly subjects), CAP might be a sentinel event for an underlying life-limiting disease.

In our study the CRB-65 score and PCT but not CRP and WBC also predicted 180-day mortality. Again, however, they performed significantly inferior to MR-proAVP and CT-proANP. These results suggest that MR-proANP and CT-proAVP are surrogate markers for patients with a high mortality risk at follow-up. There are some mechanisms that might be responsible for the marked increase in MR-proANP and CT-proAVP levels in patients with CAP. First, sepsis is the main cause of death in the acute phase of CAP. It has been shown that MRproAVP and CT-proANP serum levels are strongly elevated in patients with septic shock and are associated with poorer survival. ${ }^{18} 25-27$ Second, MR-proAVP and CT-proANP levels are increased in patients with cardiac failure and renal disease. ${ }^{25} 2628$ In patients with CAP, cardiac failure and consecutive elevation of MR-proAVP and CT-proANP levels might be due to underlying pre-existing cardiac disease or septic cardiomyopathy. As we have shown, the prognostic value of MR-proAVP and CT-proANP was independent of a pre-existing diagnosis of chronic heart failure or other chronic heart disease. In the setting of acute CAP, underlying previously unknown cardiovascular or renal disease may be aggravated by acute inflammatory activation. Although the underlying disease might not appear directly clinically evident, it could result in higher serum levels of MR-proANP and CTproAVP. This hypothesis is supported by our finding that MRproANP and CT-proAVP were stronger predictors of the risk of mortality than a history of congestive heart failure or other comorbidities alone. Taken together, the increase in MR-proANP and CT-proAVP levels might be attributable to hidden cardiovascular comorbidities or to cardiovascular dysfunction as a consequence of the acute infection.

Whether the better predictive potentials of MR-proANP and CT-proAVP for short- and long-term mortality translate into clinically relevant prediction tools-particularly of long-term mortality from CAP-remains to be determined. We recently showed that clinical scores and biomarkers can both be merged and result in superior predictions of short-term mortality. ${ }^{12}$ However, our results need prospective validation in an independent patient cohort before they can be implemented in clinical practice.

The present study has some limitations. The measurement of MR-proANP and CT-proAVP is potentially influenced by other factors such as sex, age and renal function which are known to affect brain natriuretic peptide. ${ }^{29}$ Indeed, we observed a significant correlation of MR-proANP and CT-proAVP with serum osmolarity and serum urea levels. Interpretation is difficult because the observed elevations in MR-proANP and CT-proAVP might in part be a result of renal failure related to severe CAP. Patients were not strictly consecutively included in the study. However, it was an observational study and not an interventional study, so this should not have a significant impact on the findings. The timing of biomarkers was not standardised. Blood samples were taken as soon as possible after inclusion in the study. Because of the large scale and design of the CAPNETZ study, the kinetics of the biomarkers have not been studied. However, the assessment of pneumonia severity and obtaining serum samples for biomarkers were performed concurrently. An analysis of the causes of death could not be done because the causes of death at long-term follow-up could not be consistently identified.

In conclusion, MR-proANP and CT-proAVP are strong predictors of 28-day and 180-day mortality from CAP. They were superior to the clinical CRB-65 score and the inflammatory markers PCT, CRP and WBC. If our findings are confirmed in future prospective intervention studies, MR-proANP and CTproAVP might become useful additional prognostic markers for short-term and long-term risk assessment in CAP. They may be valuable tools for assessing both patients with increased shortterm risk of death and patients at high risk of death within 180 days after pneumonia. In these patients, increased attention to possible cardiovascular disease, chronic lung disease and cancer and closer medical follow-up may be indicated.

Acknowledgements The authors are grateful to the members of the CAPNETZ Study Group: Markus Becker, Antje Kuhnke, Hartmut Lode, Malina Schmidt-loanas, Norbert Suttorp (Berlin); Torsten Bauer, Santiago Ewig, Barbara Schlosser (Bochum); Matthias Pletz, Tobias Welte (Hannover); Klaus Dalhoff, Sven Pischke, Niels Schübel (Lübeck); Ingrid Huntemann, Joachim Lorenz (Lüdenscheid); Thomas Klante (Magdeburg); Tom Schaberg, Konstanze Voigt (Rotenburg); Stefan Krüger, Christian Schumann (Ulm); Berthold Jany, Uwe Ziegler (Würzburg); Torsten Illmann, Michael Wallner, Michael Weber (IT); Heike von Baum, Susanne Gonschior, Klaus Richter (main office); and all study nurses. The authors also wish to thank Angelina Herzberg, Johanna Bogusch, Barbara Schäffus and Anne Schmiedel for their technical assistance.

Funding This study was supported by the German Federal Ministry of Education and Research (Bundesministerium für Bildung und Forschung, BMBF) grants 01 KI0103-105, Competence Network CAPNETZ.

Competing interests $\mathrm{JK}$ and $\mathrm{OH}$ are employees of BRAHMS AG, the manufacturer of the assays BRAHMS MR-proANP LIA and BRAHMS CT-proAVP LIA (Hennigsdorf, Germany). JK and $\mathrm{OH}$ do not own stock or options in the company. TW received funds for speaking at symposia organised on behalf of BRAHMS AG. All other authors have none to declare.

Ethics approval This study was conducted with the approval of the University of Magdeburg. Written informed consent was obtained from every patient prior to inclusion in the study. The study was approved by the local ethical committee.

Provenance and peer review Not commissioned; externally peer reviewed.

\section{REFERENCES}

1. Welte T, Suttorp N, Marre R. CAPNETZ-community-acquired pneumonia competence network. Infection 2004;32:234-8.

2. Almirall J, Bolibar I, Vidal $\mathrm{J}$, et al. Epidemiology of community-acquired pneumonia in adults: a population-based study. Eur Respir J 2000;15:757-63.

3. Fine MJ, Auble TE, Yealy DM, et al. A prediction rule to identify low-risk patients with community-acquired pneumonia. N Engl J Med 1997;336:243-50.

4. Lim WS, van der Eerden MM, Laing R, et al. Defining community acquired pneumonia severity on presentation to hospital: an international derivation and validation study. Thorax 2003;58:377-82

5. Capelastegui A, Espana PP, Quintana JM, et al. Validation of a predictive rule for the management of community-acquired pneumonia. Eur Respir J 2006;27:151-7.

6. Ewig S, Torres A, Woodhead M. Assessment of pneumonia severity: a European perspective. Eur Respir J 2006;27:6-8.

7. Buising $\mathbf{K L}$, Thursky KA, Black JF, et al. A prospective comparison of severity scores for identifying patients with severe community acquired pneumonia: reconsidering what is meant by severe pneumonia. Thorax 2006;61:419-24.

8. Waterer GW, Kessler LA, Wunderink RG. Medium-term survival after hospitalization with community-acquired pneumonia. Am J Respir Crit Care Med 2004;169:910-4.

9. Brancati FL, Chow JW, Wagener MM, et al. Is pneumonia really the old man's friend? Two-year prognosis after community-acquired pneumonia. Lancet 1993;342:30-3.

10. Mortensen EM, Kapoor WN, Chang CH, et al. Assessment of mortality after longterm follow-up of patients with community-acquired pneumonia. Clin Infect Dis 2003;37:1617-24.

11. Koivula I, Sten M, Makela P. Prognosis after community-acquired pneumonia in the elderly: a population-based 12 year follow-up study. Arch Intern Med 1999:159:1550-5.

12. Krüger S, Ewig S, Marre $\mathrm{R}$, et al. Procalcitonin predicts patients at low risk of death from community- acquired pneumonia. Eur Respir J 2008;31:349-55

13. Krüger S, Papassotiriou J, Marre R, et al. Pro-atrial natriuretic peptide and provasopressin to predict severity and prognosis in community-acquired pneumonia. Int Care Med 2007:33:2069-78

14. Bauer TT, Ewig S, Marre R, et al. CRB-65 predicts death from community-acquired pneumonia. J Intern Med 2006;260:93-101.

15. Mauch H, Marklein G, Kühnen E. Infektionen der tiefen Atemwege. In: Mauch H, Lütticken R, Gatermann S. eds. Quality standards for microbiological diagnostic techniques for infectious diseases. München: Urban \& Fischer Verlag, 2000 
16. Morgenthaler NG, Struck J, Christ-Crain M, et al. Pro-atrial natriuretic peptide is a prognostic marker in sepsis, similar to the APACHE II score: an observational study. Crit Care 2003;9:R37-45.

17. Morgenthaler NG, Struck J, Alonso C, et al. Assay for the measurement of copeptin, a stable peptide derived from the precursor of vasopressin. Clin Chem 2006:52:112-9.

18. Struck J, Morgenthaler NG, Bergmann A. Copeptin, a stable peptide derived from the vasopressin precursor, is elevated in serum of sepsis patients. Peptides 2005:26:2500-4.

19. Morgenthaler NG, Struck J, Thomas B, et al. Immunoluminometric assay for the midregion of pro-atrial natriuretic peptide in human plasma. Clin Chem 2004:50:234-36.

20. Niederman MS, Mandell LA, Anzueto A, et al. Guidelines for the management of adults with community-acquired pneumonia. Diagnosis, assessment of severity, antimicrobial therapy, and prevention. Am J Respir Crit Care Med 2001;163:1730-54.

21. Müller B, Suess E, Schuetz P, et al. Circulating levels of pro-atrial natriuretic peptide in lower respiratory tract infection. J Intern Med 2006;260:568-76.

22. Prat C, Lacoma A, Dominguez J, et al. Midregional pro-atrial natriuretic peptide as a prognostic marker in pneumonia. J Infect 2007:55:400-7.
23. Mortensen EM, Coley CM, Singer DE, et al. Causes of death for patients with community-acquired pneumonia: results from the Pneumonia Patient Outcomes Research Team cohort study. Arch Intern Med 2002;162:1059-64.

24. Yende S, D’Angelo G, Kellum JA, et al. Inflammatory markers at hospital discharge predict subsequent mortality after pneumonia and sepsis. Am J Respir Crit Care Med 2008:177:1242-7.

25. Jochberger S, Morgenthaler NG, Mayr VD, et al. Copeptin and arginine vasopressin concentrations in critically ill patients. J Clin Endocrinol Metab 2006;91:4381-6.

26. Jochberger S, Mayr VD, Luckner G, et al. Serum vasopressin concentrations in critically ill patients. Crit Care Med 2006;34:293-9.

27. Witthaut R, Busch C, Fraunberger $P$, et al. Plasma atrial natriuretic peptide and brain natriuretic peptide are increased in septic shock: impact of interleukin-6 and sepsisassociated left ventricular dysfunction. Intensive Care Med 2003;29:1696-702.

28. Gegenhuber A, Struck J, Poelz W, et al. Midregional pro-A-type natriuretic peptide measurements for diagnosis of acute destabilized heart failure in short-of-breath patients: comparison with B-type natriuretic peptide (BNP) and amino-terminal proBNP. Clin Chem 2006;52:827-31.

29. McLean AS, Huang SJ, Nalos M, et al. The confounding effects of age, gender, serum creatinine, and electrolyte concentrations on plasma B-type natriuretic peptide concentrations in critically ill patients. Crit Care Med 2003:31:2611-8.

\section{Pulmonary puzzle}

\section{Three diagnoses become one: a woman with ground-glass attenuation develops fever}

\section{CLINICAL PRESENTATION}

In 2002 a 73-year-old woman (non-smoking, medical history of allergic rhinitis) was referred to a rheumatologist because of synovitis of the metacarpophalangeal and proximal interphalangeal joints of both hands which had slowly developed over 3 months. Late-onset rheumatoid arthritis was suspected. Morning stiffness, rheumatoid nodules, erosions of cartilage or bone, rheumatoid factor and antibodies to cyclic citrullinated peptides were absent. Prednisone (2.5-5 mg daily) and nonsteroidal anti-inflammatory drugs were started.

In 2004 she was referred to a respiratory physician for analysis of a persistent dry cough and pleural effusion. CT scans of the lungs (six scans between 2004 and 2009, see representative scan in figure 1 revealed fleeting bilateral multifocal consolidations with ground-glass attenuation. Bronchoscopic examination was normal. The lavage fluid showed 39\% macrophages, 58\% lymphocytes and $2 \%$ neutrophils. Diffusing capacity (transfer factor) was low while spirometry was normal. These findings were interpreted as lung disease secondary to rheumatoid arthritis. Because polyarthritis had responded to low-dose glucocorticoid treatment and the pulmonary lesions appeared stable over time, this low-dose regimen was maintained over the years.

In 2005 diabetes mellitus type 2 was diagnosed which was treated with oral blood glucose lowering agents (metformin $500 \mathrm{mg}$ twice daily) resulting in adequate control (glycosylated haemoglobin $7.1 \%$ ). No diabetic retinopathy was present. In 2006 she developed painful polyneuropathy. The neurologist ascribed polyneuropathy to diabetes mellitus and started amitriptyline. No further tests were ordered.

In 2008 she developed persistent fevers, elevated inflammatory parameters (peak erythrocyte sedimentation rate $106 \mathrm{~mm} / \mathrm{h}$, C-reactive protein $135 \mathrm{mg} / \mathrm{l}$ ) and normocytic anaemia (haemoglobin $8.7 \mathrm{~g} / \mathrm{dl}(5.3 \mathrm{mmol} / \mathrm{l}))$. Elaborate testing failed to provide new insights. These tests included serial blood cultures, tests for tuberculosis, syphilis, borrelia, hepatitis, HIV, bone marrow biopsy, CT scans of chest and abdomen, positron emission tomography, leucocyte scan, bronchoalveolar lavage and temporal artery biopsy.

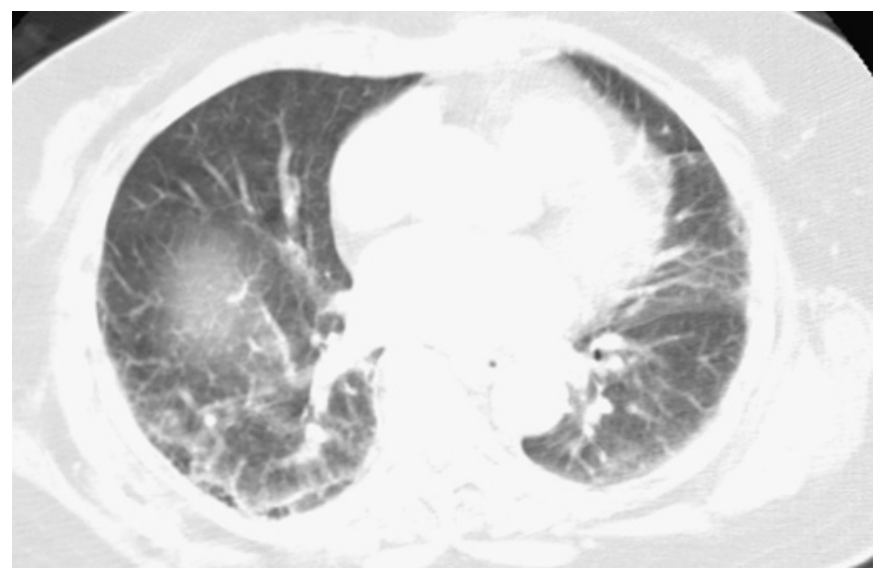

Figure 1 CT scan of the lungs of a 73-year-old woman with groundglass attenuation and fever.

\section{QUESTION}

A woman with presumed rheumatoid arthritis, associated lung disease, diabetes mellitus and polyneuropathy develops fevers of unknown origin. Which alternative diagnosis is possible based on the clinical information and the CT image (figure 1) and which tests would you order to confirm this?

See page 270 for answer

\section{Ewout J Hoorn, ${ }^{1}$ Jan A van Laar, ${ }^{1}$ Jan $\mathbf{G}$ den Hollander, ${ }^{2}$ Johan M Kros, ${ }^{3}$ Esther Brusse ${ }^{4}$}

${ }^{1}$ Department of Internal Medicine, Erasmus Medical Center, Rotterdam, The Netherlands; ${ }^{2}$ Department of Internal Medicine, Maasstad Ziekenhuis, Rotterdam, The Netherlands; ${ }^{3}$ Department of Pathology, Erasmus Medical Center, Rotterdam, The Netherlands; ${ }^{4}$ Department of Neurology, Erasmus Medical Center, Rotterdam, The Netherlands

Correspondence to Dr Ewout J Hoorn, Department of Internal Medicine, Erasmus Medical Center, P 0 Box 2040, Room D-405, 3000 CA Rotterdam, The Netherlands; ejhoorn@gmail.com

\section{Competing interests None.}

Patient consent Obtained.

Provenance and peer review Not commissioned; externally peer reviewed

Thorax 2010:65:214. doi:10.1136/thx.2009.128397 